\title{
Mixtures of endocrine-disrupting contaminants induce adverse developmental effects in preweaning rats
}

\author{
Marta Axelstad, Sofie Christiansen, Julie Boberg, Martin Scholze ${ }^{1}$, Pernille Rosenskjold Jacobsen, \\ Louise Krag Isling, Andreas Kortenkamp ${ }^{1}$ and Ulla Hass \\ Division of Toxicology and Risk Assessment, National Food Institute, Technical University of Denmark, \\ Mørkhøj Bygade 19, DK-2860 Søborg, Denmark and ${ }^{1}$ Institute for the Environment, Brunel University, \\ Kingston Lane, Uxbridge, Middlesex UB8 3PH, UK \\ Correspondence should be addressed to M Axelstad; Email: maap@food.dtu.dk
}

\begin{abstract}
Reproductive toxicity was investigated in rats after developmental exposure to a mixture of $\mathbf{1 3}$ endocrine-disrupting contaminants, including pesticides, plastic and cosmetic ingredients, and paracetamol. The mixture was composed on the basis of information about high-end human exposures, and the dose levels reflecting 100, 200, and 450 times this exposure were tested. The compounds were also grouped according to their estrogenicity or anti-androgenicity, and their joint effects were tested at two different doses, with each group reflecting 200 or 450 times human exposure. In addition, a single paracetamol dose was tested ( $350 \mathrm{mg} / \mathrm{kg}$ per day). All exposures and a vehicle were administered by oral gavage to time-mated Wistar dams rats throughout gestation and lactation, and their offspring were assessed for reproductive effects at birth and in prepuberty. The mixture doses, which included the anti-androgenic compounds, affected the male offspring by causing decreased anogenital distance, increased nipple retention (NR), and reduced ventral prostate weights, at both medium and high doses. In addition, the weights of the levator ani/bulbocavernosus muscle (LABC) were decreased at the high dose of anti-androgen mixture. No effects were seen after exposure to the estrogenic chemicals alone, whereas males exposed solely to paracetamol showed decreased LABC weights and increased NR. Thus adverse reproductive effects were observed at mixtures reflecting 200 times high-end human exposure, which is relatively close to the safety margin covered by the regulatory uncertainty factor of 100. This suggests that highly exposed human population groups may not be sufficiently protected against mixtures of endocrine-disrupting chemicals.

Reproduction (2014) 147 489-501
\end{abstract}

\section{Introduction}

There is good evidence from animal studies that developmental exposure to environmental agents with endocrine-disrupting properties can cause adverse reproductive effects, including decreased anogenital distance (AGD), increased nipple retention (NR), altered weights and histopathology of reproductive organs, and

\footnotetext{
This paper forms part of a special issue of Reproduction on Endocrine Disrupters. This article was presented at the 7 th Copenhagen Workshop on Endocrine Disrupters, 28-31 May 2013. The meeting was supported by the Danish Ministry of the Environment - Environmental Protection Agency as an activity under the Danish Centre on Endocrine Disrupters. Publication of this special issue has been supported by the Society for Reproduction and Fertility. The opinions or views expressed in this special issue are those of the authors, and do not necessarily reflect the opinions or recommendations of the Danish Ministry of the Environment - Environmental Protection Agency or the Society for Reproduction and Fertility. The Guest Editors for this special issue were Anna-Maria Andersson, Hanne Frederiksen, Niels Erik Skakkebæk, Rigshospitalet, Denmark, Kenneth M Grigor, Western General Hospital Edinburgh, UK and Jorma Toppari, University of Turku, Finland.
}

reduced semen quality (Hotchkiss et al. 2008, Christiansen et al. 2009, 2010, Axelstad et al. 2011, Hass et al. 2012, Jacobsen et al. 2012). The environmental agents that cause these effects often have many modes of action, but for simplicity, they are here described as being estrogenic or anti-androgenic. For both classes of compounds, it has also been shown that they can act together to produce effects at doses that individually are not associated with any observable responses (Silva et al. 2002, Hass et al. 2007, 2012, Metzdorff et al. 2007, Howdeshell et al. 2008, Rider et al. 2008, 2010, Christiansen et al. 2009, Jacobsen et al. 2012). A majority of in vivo mixture studies have tested the combinations of anti-androgenic compounds and although the doses applied in these experiments have been in the range of no-observed-adverse-effect levels (NOAELs), they were still quite far from environmental exposures experienced by humans. Furthermore, those studies have primarily been designed to explore the predictability of mixture effects, by using various assessment concepts, rather than to investigate environmentally relevant combinations. 
Based on this lack of knowledge about effects caused by environmentally relevant contaminant exposures, we have previously performed an exploratory in vivo study in rats, with a mixture of 13 endocrine-disrupting chemicals, modeled on information about environmental exposures in humans (Christiansen et al. 2012). As described in more detail in Christiansen et al. (2012), the chemicals for the mixture were selected based on information about their endocrine-disrupting effects in vivo and available data about human exposures, to guide the choice of doses to be combined in the mixture. Other chemicals could not be included in the mixture because either their effects or their human exposures were not known, and this has constrained somewhat the selection of chemicals for the mixtures investigated in this study. Of the 13 selected chemicals, eight had predominantly anti-androgenic properties. These included the two phthalates - di- $n$-butyl phthalate (DBP) and di-(2-ethylhexyl) phthalate (DEHP), the five pesticides - vinclozolin, prochloraz, procymidone, linuron, epoxiconazole, and the DDT metabolite $p, p^{\prime}$-DDE. The mixture also included four predominantly estrogenic substances, the two u.v.-filters octyl methoxycinnamate $(\mathrm{OMC})$ and 4-methyl-benzylidene camphor (4-MBC), the phenolic compound bisphenol A (BPA), and the preservative butyl paraben (BP). Furthermore, the mixture included the analgesic drug paracetamol, which has previously been shown to act as an antiandrogen (Kristensen et al. 2011, 2012). Table 1 gives an overview of the uses of these chemicals, their predominant modes of action in causing reproductive tract alterations in experimental animals, some of the relevant effects seen in rodents, together with the NOAELs and lowest-observed-adverse-effect levels (LOAELs) reported in the literature for these effects. More information about each of the chemicals and the rationale for selecting these 13 chemicals for the mixture are presented in detail in Christiansen et al. (2012).

We used information about the potency of the selected chemicals to predict the effects of the mixture by using the point-of-departure index (PODI). This cumulative risk assessment method assumes that the joint action of mixture components can be approximated by dose addition, and that antagonisms or synergisms are not relevant (Christiansen et al. 2012), a conjecture that is supported by empirical evidence (Hass et al. 2007, Howdeshell et al. 2008, Rider et al. 2008, 2010, Christiansen et al. 2009). The method uses NOAELs or benchmark doses of the individual components as input values, together with the dose of each component present in the mixture. Assuming that all mixture components act together according to dose addition, significant combination effects are only expected to occur if the PODI is $>1$. The PODI for the combination of the 13 adjusted human high exposure estimates summed up to 0.016 (Table 2). This means that a mixture of dose $1.12 \mathrm{mg} / \mathrm{kg}$ per day, equal to the sum of the adjusted high-end human intakes, was not expected to produce any endocrinedisrupting effects in the rat. Even an increase in all individual doses by a factor of 62 , which would bring the PODI to 1, would not be expected to produce observable joint effects. In the previous exploratory study, the primary focus was to investigate how developmental exposure to mixtures of all chosen chemicals (at doses equivalent to 150 - and 450-fold high-end human exposures) affected early markers of endocrine disruption (Christiansen et al. 2012), and to investigate reproductive effects in adult and senescent offspring (Isling et al. 2013). These doses may appear quite high at first glance, but are indeed highly relevant considering the known differences in toxicokinetics between rat and humans. It is widely accepted that higher doses need to be administered to rats to achieve comparable effects, and this is the basis for using default uncertainty factors of 100 for the extrapolation of effects in rats to humans. The study showed that even the low mixture dose (TotalMix150) caused some signs of anti-androgenic effects, as the numbers of retained nipples in the male offspring was slightly but significantly elevated compared with untreated controls. As anticipated on the basis of the PODI calculations, the male offspring exposed to the high-dose mixture (TotalMix450) showed anti-androgenic effects, including significantly increased NR and reduced weights of the ventral prostate. Exposure only to a mixture of the antiandrogenic components of the mixture (AAMix450) also caused increased NR, whereas no significant effects on reproductive organ weights were seen.

In this study, we expanded our previous investigation on early markers of endocrine disruption, after developmental exposure to a mixture of 13 contaminants (TotalMix), by comparing the effects of the total mixture with the effects of each of its subcomponents, i.e. the antiandrogens (AAMix), the estrogens (EMix), and paracetamol (PM), separately. The statistical power was increased by including larger size groups $(n=14-20)$ than in the exploratory study, and we placed emphasis on investigating even lower human-relevant doses, and included a dose only 100 times larger than high-end human intakes. Detailed studies on the effects of endocrine-disrupting mixture on sexual maturation, mammary development, sperm count, estrous cyclicity, behavior, serum biomarkers, reproductive organ histology in males and females, and gene expression studies on prostate and mammary gland are ongoing and will be presented in other papers.

\section{Materials and methods}

\section{Test compounds}

The test compounds in this study were DBP (purity $>99.0 \%$, Cas no. 84-74-2), DEHP (purity $>99.5 \%$, Cas no. 117-81-7), vinclozolin (purity $>99.5 \%$, Cas no. 50471-44-8), prochloraz (purity $>98.5 \%$, Cas no. 67747-09-5), procymidone 


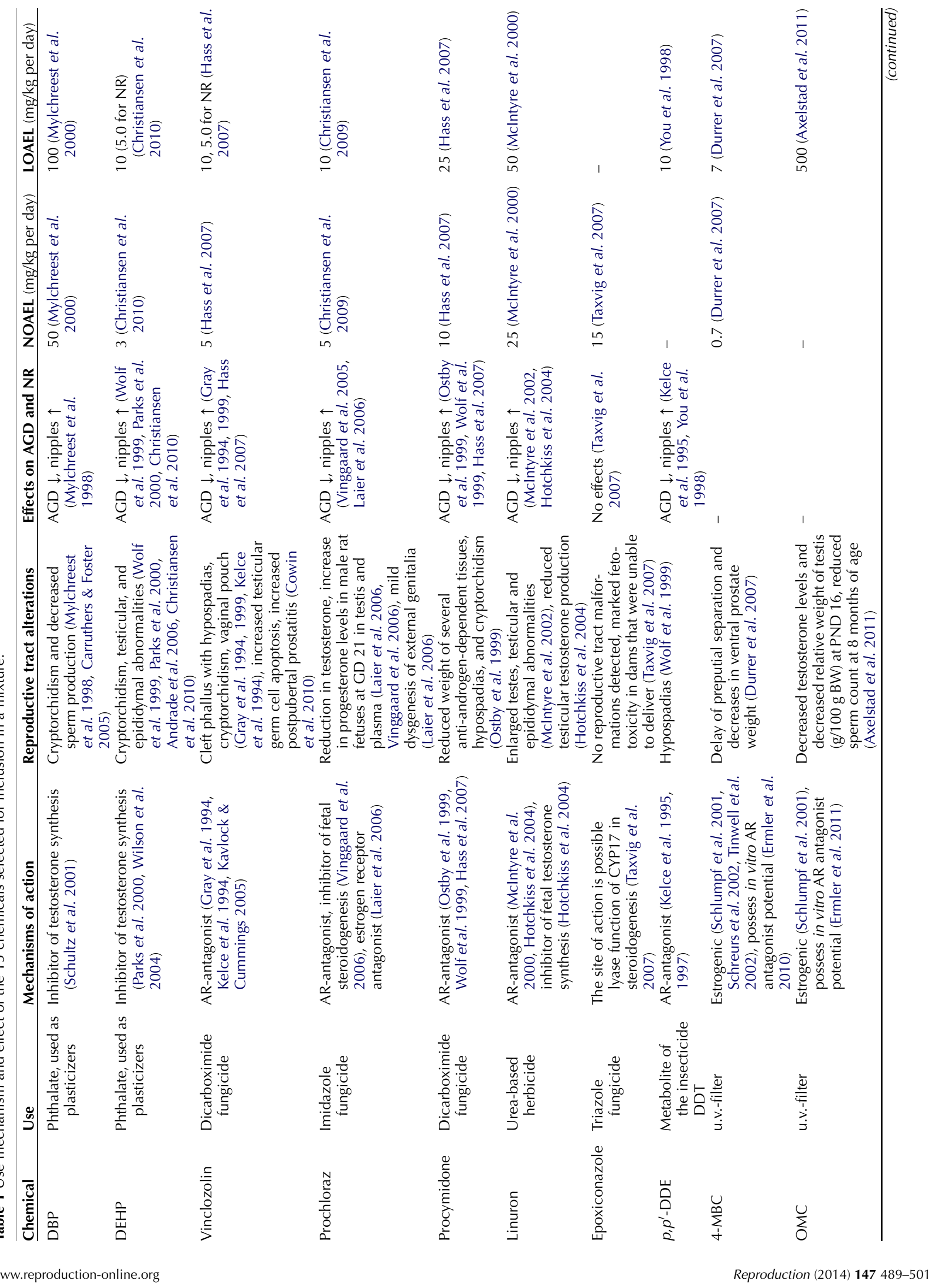




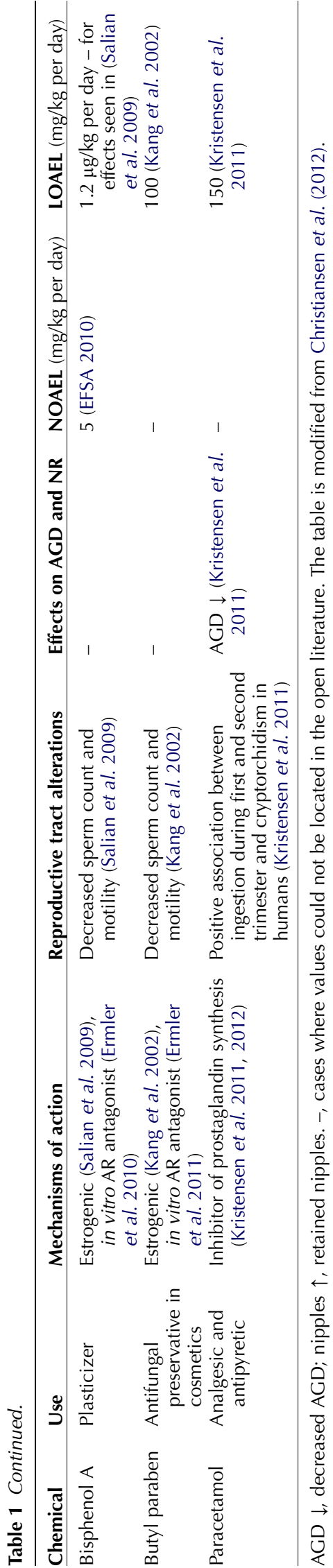

(purity $>99.5 \%$, Cas no. 32809-16-8), linuron (purity $>99.0 \%$, Cas no. 330-55-2), epoxiconazole (purity $>99.0 \%$, Cas no. 106325-08-8), OMC (purity $>98.0 \%$, Cas no. 5466-77-3), $p, p^{\prime}$-DDE (purity $>98.5 \%$, Cas no.72-55-9) were purchased from VWR - Bie and Berntsen (Herlev, Denmark). While 4-MBC (purity $>98.0 \%$, Cas no. 36861-47-9), BPA (purity $>99.5 \%$, Cas no. $80-05-7$ ), BP (purity $>99.0 \%$, Cas no. 94-26-8), and paracetamol (purity $>99.0 \%$, Cas no. 103-90-2) were purchased from Sigma-Aldrich, corn oil, which was used both as a control compound and as a vehicle, was purchased from VWR - Bie and Berntsen.

\section{Animals and exposure}

A total of 156 time-mated nulliparous, young adult Wistar rats (HanTac:WH, SPF, Taconic Europe, Ejby, Denmark) were supplied at gestation day 3 (GD 3) of pregnancy. The study was performed using four blocks of 38-40 dams (separated by 1 week), and all groups were equally represented in the blocks. Animal experiments were carried out at the DTU National Food Institute (Mørkhøj, Denmark) facilities. Ethical approval was obtained from the Danish Animal Experiments Inspectorate. The authorization number is: 2012-15-2934-00089 C4. The experiments were overseen by the National Food Institutes in-house Animal Welfare Committee for animal care and use.

The animals were housed in pairs until GD 17 and alone thereafter under standard conditions in semi-transparent polycarbonate type III cages (1291H Eurostandard Type III, Tecniplast) $(15 \times 27 \times 43 \mathrm{~cm})$ with Aspen wood chip bedding (Tapvei, Gentofte, Denmark), Enviro-Dri nesting material (Brogaarden, Lynge, Denmark), and plastic shelters (Brogaarden). They were housed in an animal room with controlled environmental conditions with a $12 \mathrm{~h}$ light: $12 \mathrm{~h}$ darkness cycle with 500 lux light intensity starting at $2100 \mathrm{~h}$, humidity $55 \% \pm 5$, temperature at $21 \pm 1{ }^{\circ} \mathrm{C}$, and ventilation, changing air ten times per hour. All animals were fed on a standard diet with ALTROMIN 1314 (soy- and alfalfa-free, ALTROMIN $\mathrm{GmbH}$, Lage, Germany). Acidified tap water (to prevent microbial growth) in polycarbonate bottles (Tecniplast) was provided ad libitum.

On the day after arrival (GD 4), the time-mated dams were pseudo-randomly distributed into nine groups with similar body weight (BW) distributions. The dams received vehicle (controls), or one of the eight mixtures presented in Table 2. These included three doses of total mixture (TotalMix100, TotalMix200, TotalMix450), two doses of anti-androgens only (AAMix200, AAMix450), two doses of estrogens only (EMix200, EMix450), and paracetamol (PM). As can be seen in Table 3, 16 and 20 mated dams were used in each dose group, resulting in 14 and 20 viable litters per group. Dams that did not give birth were omitted from the experiment. Mixtures and vehicle were administered by oral gavage with a stainless steel probe $1.2 \times 80 \mathrm{~mm}$ (Scanbur, Karlslunde, Denmark) from GD 7 to the day before expected birth (GD 21) and again after birth from PND 1 to PND 22 to cover the most sensitive windows of reproductive development in rat offspring. The day the vaginal plug was detected was designated as GD 1 and the expected day of delivery (GD 23) was designated as pup day (PD) 1. 


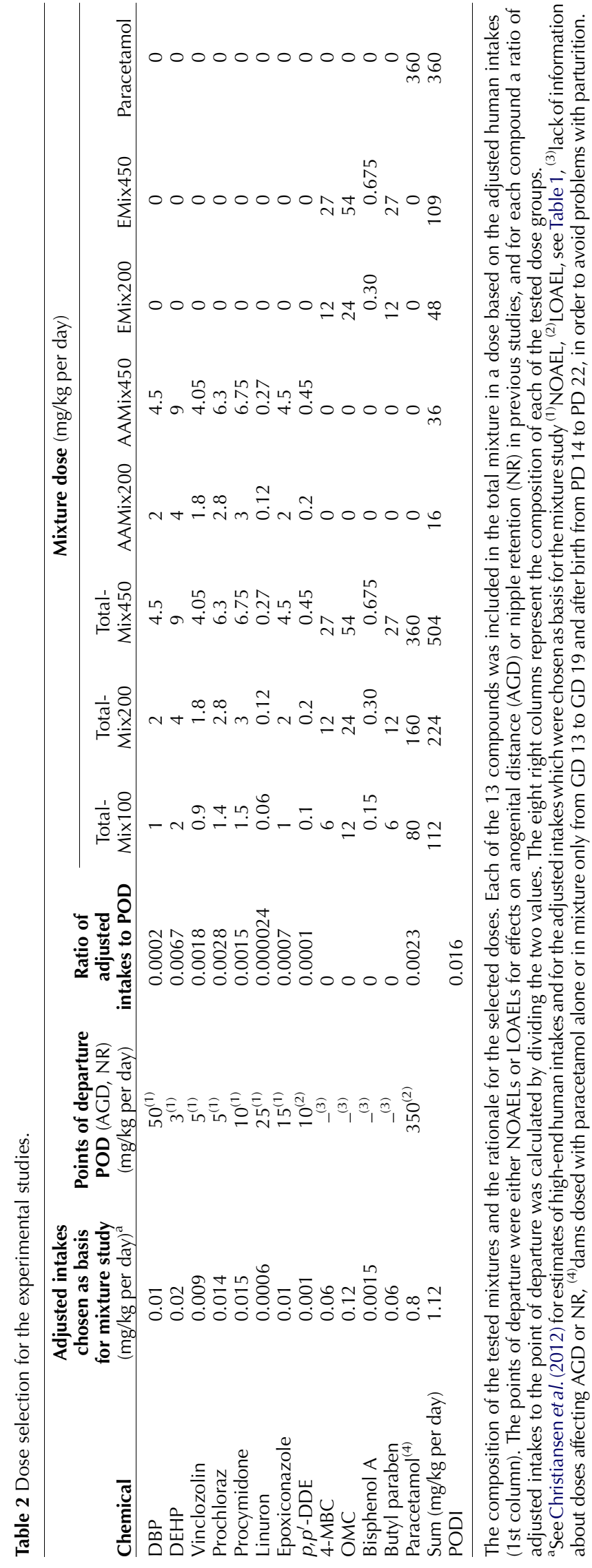

In the gestation period, only PM was added to the mixture groups and dosed to the PM group from GD 13 to GD 19. The later initiation of $\mathrm{PM}$ exposure during pregnancy, i.e. GD 13 instead of GD 7, was chosen to avoid potential effects on implantation of the embryos (Gupta et al. 1981). The termination of paracetamol dosing at GD 19 instead of GD 21 was aimed at avoiding potential effects on the ability of the dams to give birth. In the postnatal period, dams were again exposed to paracetamol from PD 14 to PD 22, as this was relevant for blood measurements of chemicals (reported elsewhere). Dose administration to the animals was done by a qualified animal technician. All doses were given in vehicle $(2 \mathrm{ml} / \mathrm{kg})$ via oral gavage during morning hours. The stock solutions were prepared by a technician just before the study was carried out and during exposure period the solutions were stored in the animal rooms. The dams were inspected twice a day for general toxicity, including changes in clinical appearance (e.g. sedation and tremor). BWs were recorded on GD 4 and daily during the dosing period to monitor a decrease or increase in weight gain and to adjust dose according to the weight. BWs of dam were used to calculate BW gain during pregnancy and maternal weight gain from start of dosing (GD 7) till the day post delivery (PD 1).

\section{Pregnancy and postnatal development}

Gestation length and body weight of dams and of individual pups were recorded after delivery in all pregnant animals. The pups were counted, sexed, and checked for anomalies. The dead pups were macroscopically investigated for changes when possible. The BW of offspring was recorded again on PD 6 and 13, and the litter size and pup survival were registered.

After weaning of the offspring at PD 22, the dams were killed and the number of implantation sites was registered to calculate postimplantation and perinatal loss.

\section{$A G D$ and $N R$}

AGD measurements in all offspring were performed by the same skilled technician, who was blinded with regard to exposure group. Measurements were performed using an ocular stereomicroscope with unit markings on the ocular. In male offspring the measured AGDs were in the range of 17-27 units (i.e. $2.81-4.46 \mathrm{~mm}$ ) and in female offspring the AGDs were in the range of 8-16 units (i.e. 1.32-2.64 mm). On PD 13, all male and female pups were examined for NR; nipple buds are located in female offspring. The registration criterion used for assessing NR on the whole data set was less sensitive in this than the one used in the exploratory study, as only nipple buds that could be felt on the skin of the offspring were registered. For comparison with the previous study, all pups from blocks 3 and 4 were additionally assessed for the presence of areolas (dark focal area that did not necessarily have a nipple bud that could be felt through the skin), and then dichotomised in terms of presence or absence of NR, i.e. a yes or no answer for each pup.

\section{Necropsy PD 16/17}

On PD 16, one male pup and, on PD 17, one female pup per litter were weighed (BW) and decapitated. Malformations of 


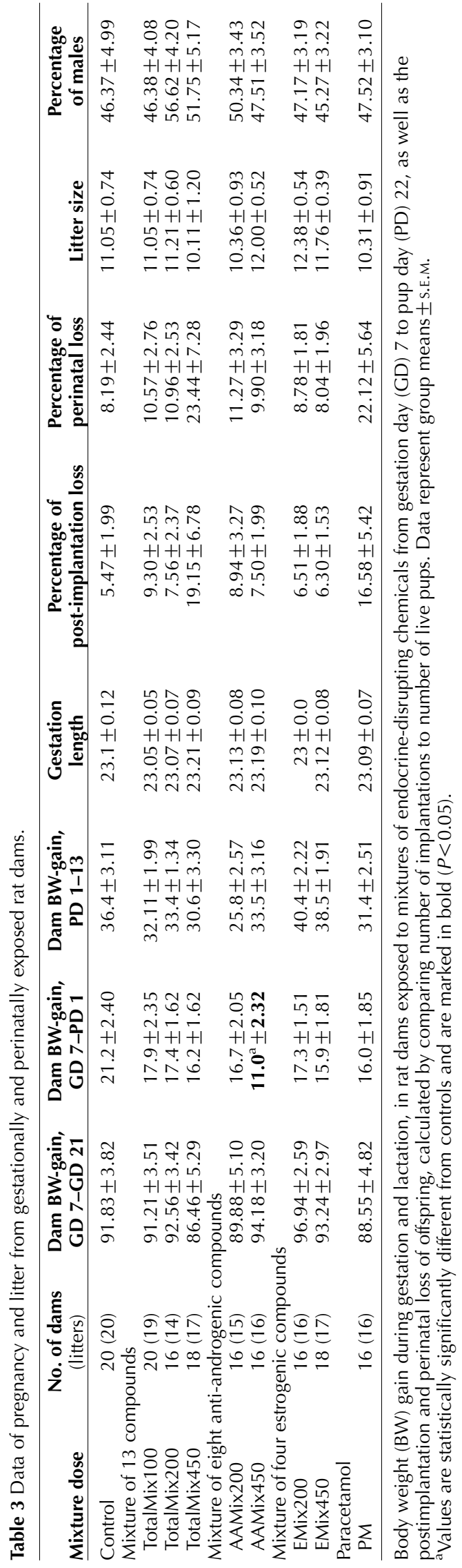

external genitals in males were scored (score 0-3) (as described in Metzdorff et al. (2007)). In males, alternately the right and the left testes, both epididymides, ventral prostate, seminal vesicles, levator ani/bulbocavernosus muscle (LABC), and bulbouretral gland were excised and weighed. Male thyroid glands were excised and thyroid glands from the TotalMix450 and controls group were fixed in formalin, routinely processed for paraffin embedding, stained with hematoxylin and eosin, and examined histopathologically. Changes related to follicular cell hyperplasia and hypertrophy were scored. In females, one ovary, uterus, and thyroid were excised and weighed.

\section{Statistical analysis}

Data from continuous endpoints were examined for normal distribution and homogeneity of variance, and if relevant, transformed. AGD data were analyzed using pups' birth weights as covariate and by the AGD-index, i.e. AGD divided by the cube root of BW. The cube root transformation was used because it improves the comparison between the three-dimensional end point weight and the one-dimensional AGD (Gallavan et al. 1999, Gray et al. 1999). When more than one pup from each litter was examined, statistical analyses were adjusted using litter as an independent, random, and nested factor. Organ weights were analyzed using BW as a covariate. Fisher's exact test was used for statistical evaluation of thyroid histopathology. The number of nipples (NR) was analyzed together for all four blocks and was assumed to follow a binomial distribution with a response range between 0 and $\theta_{\max }$, with $\theta_{\max }$ being equal to the biologically possible maximal number of nipples in rats, either 12 or 13 . The choice of $\theta_{\max }$ was decided by considering the global fit (information criterion of Schwarz). Litter effects on NR and overdispersion in the data was accounted for by using generalized estimating equation (GEE). In addition, all male pups from blocks 3 and 4 were analyzed for both nipple buds and areolas, and if present they were dichotomized as an 'all or none response' for the animal. Data were analyzed in a similar way as for NR, i.e. by quantal data analysis considering litter effects and overdispersion. Statistical significance was assessed using multiple contrast tests (two-sided Dunnett contrasts, global error rate $\alpha=5 \%$ ) (Bretz et al. 2005). These tests were chosen as they were already implemented in the SAS procedure PROC GENMOD which was used for all statistical analysis (SAS Institute, Inc., Cary, NC, USA).

\section{Results}

A statistically significant decrease in maternal weight gain was observed from GD 7 to PD 1 in the dams dosed with the highest mixture of the anti-androgens (Table 3), but no other clinical signs of general maternal toxicity were seen. Maternal weights were not significantly affected in any dose group compared with controls during the lactation period, and no differences in gestation length, post-implantation loss, litter size, sex ratio, perinatal loss, or pup survival were seen after exposure to the different mixtures (Table 3). BWs of male (Table 4) and female offspring (Supplementary Table 1, 


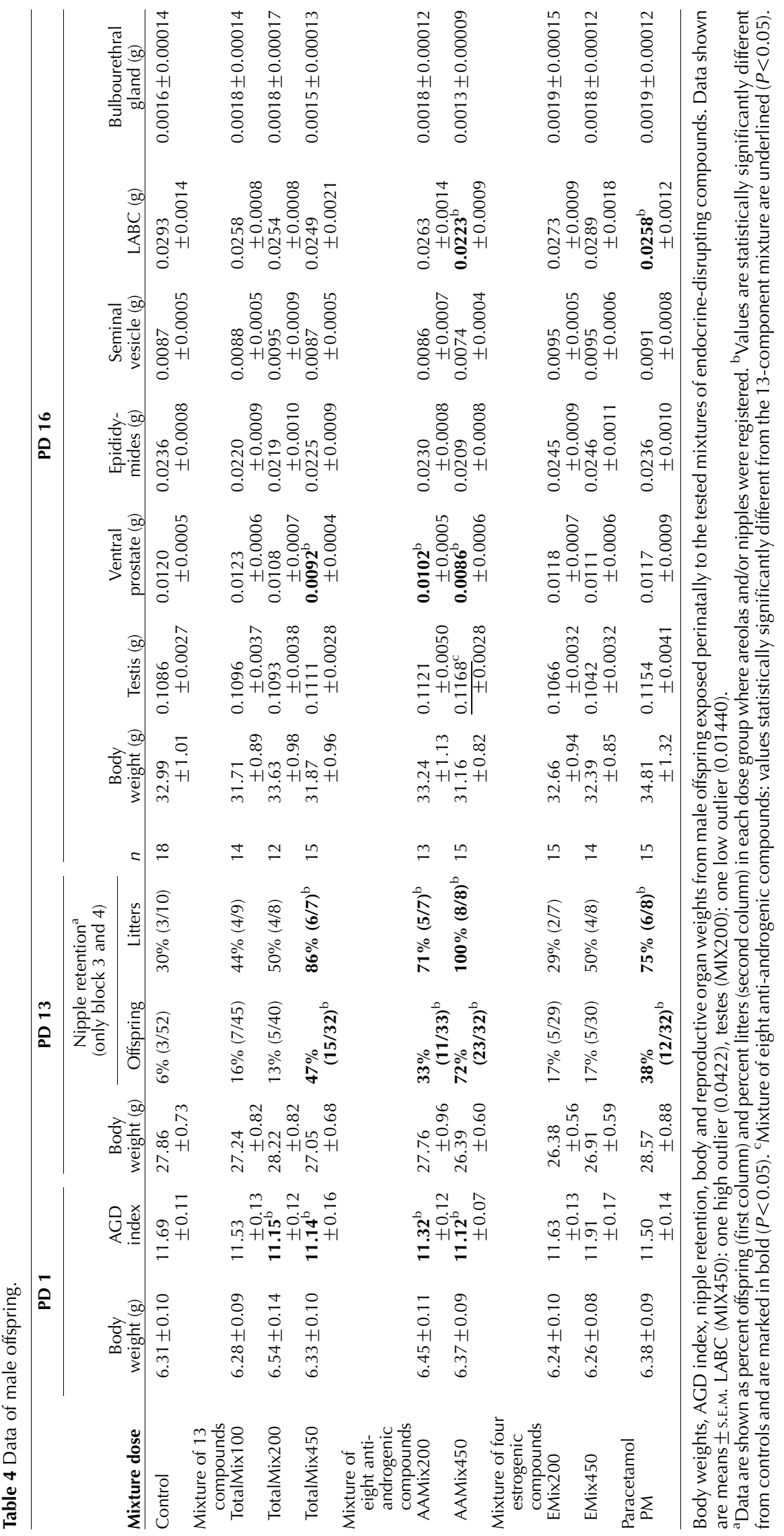


see section on supplementary data given at the end of this article) were unaffected when measured at birth and on days 6,13 , and 16/17.

A statistically significant decrease in AGD was seen in males exposed to mixtures containing anti-androgenic compounds, at median and high doses (TotalMix200, TotalMix450, AAMix-200, and AAMix450). This effect was seen both when analyzed as AGD index (AGDI), (Table 4 and Fig. 1) and when the AGDs were analyzed with the birth weight of the pups as a covariate (data not shown). For the groups exposed to the mixture of antiandrogens only, the effects were dose dependent, as the high dose caused a larger reduction than the medium dose, whereas both doses together with the estrogenic compounds reduced male AGDs similarly (TotalMix200, TotalMix450). The estrogen mixture and PM on its own did not significantly affect male AGDs. In female offspring no significant effects on AGD were seen in any dose group (Supplementary Table 1).

The registration of NR from animals in blocks 3 and 4 is shown as percent of affected offspring and percent of affected litters in each dose group (Table 4). Registration of pups with visible areolas from the last two study blocks showed a statistically significant increase in NR in the group exposed to the highest mixture of all 13 compounds (TotalMix450), the low and the high doses of the anti-androgenic mixtures (AAMix200 and 450) and to paracetamol (the PM group). When registered only as nipple buds in males from the whole data set, the number of nipples was significantly increased in the same three mixture groups as described earlier, whereas the effect was not statistically significant in the PM group (data not shown). The number of nipple buds in female offspring was not affected in any dose group (Supplementary Table 1).

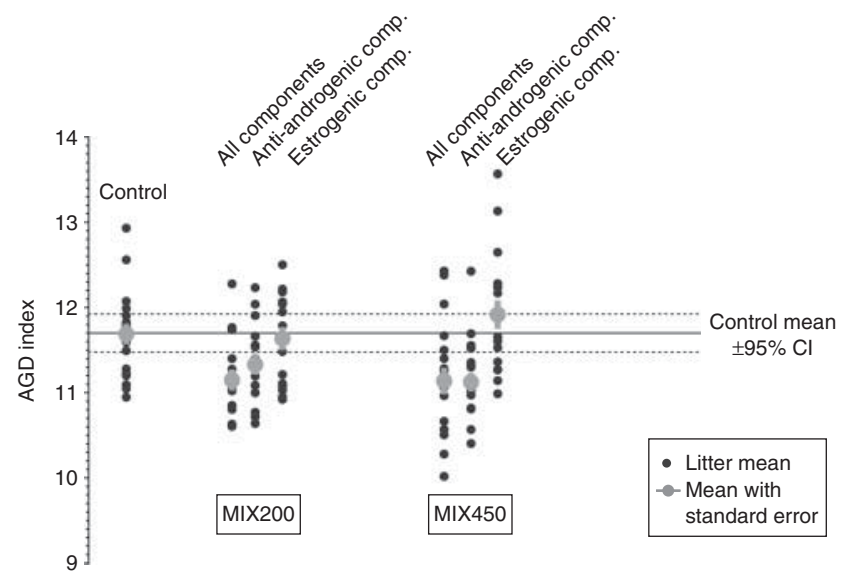

Figure 1 Anogenital index in male offspring. AGDI at birth, shown as litter means (black dots) and group means with standard errors (grey dots and bars), in offspring exposed to 200 and 450 times human exposure levels, of either all compounds (Totalmix), only antiandrogens (Amix), or only estrogens (Emix).
In PD 16, ventral prostate weights were significantly reduced in males in the TotalMix450 group and in the AAMix200 and AAMix450 groups (Table 4). The LABC weights were also significantly decreased at PD 16 (Table 4). Although a slight effect was seen in all TotalMix groups, the reduction in LABC weights was only statistically significant in the AAMix450 and the PM groups. No malformations of external male genitalia were noted. No statistically significant difference in thyroid histopathology scores was observed between males from the TotalMix450 group and controls (data not shown).

In female offspring killed at PD 17, no difference was observed for weight the of uterus or thyroid gland. Ovary weights were significantly increased in the TotalMix450 and AAMix450 groups (Supplementary Table 1), but comparison with results from the exploratory study indicated that this might be a chance finding.

\section{Discussion}

Significant effects on AGD, NR, and reproductive organ weights were seen in male offspring exposed to mixtures of the tested endocrine-disrupting chemicals. The majority of the 13 chemicals were present at doses below their NOAELs for endpoints relevant to disruption of male sexual differentiation. Our mixture study shows that combination effects can be demonstrated in the rat when endocrine-disrupting compounds are combined at doses 200-fold higher than estimated high-end human exposure levels. As seen in our previous exploratory study (Christiansen et al. 2012), the PODI approach was proved to be a surprisingly useful tool for anticipating the combined effects of environmental contaminants on rat offspring exposed during gestation and lactation.

The AGDIs in male offspring exposed to the higher doses of the TotalMix and the AAMix were significantly reduced by $3-5 \%(4.6,4.7,3.2$, and $4.9 \%$ reduction in TotalMix200, TotalMix450, AAMix200, and AAMix450 respectively). These effect magnitudes were slightly higher than those observed in the previous study, which did not reach statistical significance (AGDI reductions of 1.1, 1.5, and 3.0\% at TotalMix150, TotalMix450, and AAMix450 respectively, Christiansen et al. (2012)). These differences between the mean AGDI values in these two studies are within the range of variation normally expected from experiment to experiment (Christiansen et al. 2010). The observed reductions in AGDI seemed to be caused by the anti-androgenic compounds present in TotalMix, as the changes in these groups were similar to the reductions observed in the groups exposed to the anti-androgenic chemicals alone (AAMix). The addition of estrogenic compounds to the mixture did not seem to alter the anti-androgenic response, and exposure to the mixture of estrogens alone did not cause any significant effects on AGDs either. In other cases, estrogenic compounds may affect AGD or contribute additively to adverse effects caused by exposure to anti-androgens, 
and some of the estrogenic compounds are known to alter AGD (Boberg et al. 2013, Christiansen et al. 2013). The lack of effect of the estrogens tested in this study could very well be explained by the fact that the doses of these compounds included in the mixture were modeled on human exposure levels, rather than being added to the mixture based on their potency on a given adverse reproductive endpoint.

When the number of nipple buds was used as the endpoint in our data analysis, a significant increase in NR was seen in male offspring exposed to TotalMix450, AAMix200, and AAMix450. When we enhanced the assessment by including areolas as an additional marker for NR, all three groups were confirmed to be significantly affected, despite the fact that the number of animals available for data analysis was reduced by around the half in each treatment group. Moreover, males exposed to paracetamol showed signs of significantly increased NR (Table 4).

In previous studies, we demonstrated that exposure to paracetamol during development can adversely affect male reproductive development in rats, by reducing male AGDs (Kristensen et al. 2011). The selected dose of paracetamol corresponds to a high level of human exposure (i.e. the maximum recommended dose). Such high exposure may seem quite unlikely to occur during the long time span of almost the whole pregnancy and lactation period. On the other hand, it might not have been necessary for paracetamol exposure over such a long time period to experience its adverse reproductive effects. If paracetamol by chance is taken during the most sensitive period of fetal reproductive development, a few days or weeks may be enough to affect the reproductive function of the child later in life. In a recent paper describing endocrine changes in fetal human testis exposed to paracetamol and other painkillers ex vivo, the authors suggested a critical age window for sensitivity to these compounds (Mazaud-Guittot et al. 2013).

In this study, a paracetamol dose of $350 \mathrm{mg} / \mathrm{kg}$ per day led to significant increases in NR and reduced the weight of LABC (Table 4), whereas a reduction in AGDI by $1.6 \%$ could not be confirmed as statistically significant. This is apparently contradicting our previous study where we observed on average a $4-7 \%$ reduced AGDI in fetal rat offspring on GD 21, with the same exposure dose (Kristensen et al. 2011). This disagreement could, however, be explained in terms of a shortened exposure duration of 7 days in this study (GD 13-19), compared with 15 days in the previous one (GD 7-21) and a different age of examination. The anti-androgenic effect of paracetamol in developmentally exposed rats is corroborated by findings of reduced testosterone production in ex vivo fetal rat testes (Kristensen et al. 2011, 2012) and by in vitro findings in adult human testes (Albert et al. 2013). Furthermore, PM exposure during fetal development in humans has also been associated with increased risk of cryptorchidism in several epidemiological studies (Jensen et al. 2010, 2011a, Kristensen et al. 2011, Snijder et al. 2012), indicating that the effects seen in the rats could be indicative of adverse effects in humans. Further examination of endocrine-sensitive endpoints later in life may clarify whether this anti-androgenic effect of paracetamol causes permanent adverse reproductive effects.

The adversity of moderately shortened AGDs or of slightly increased NR in male offspring can be debated. However, as shown in previous toxicity studies, the effects on AGD and NR are highly predictive for increased risk of adverse reproductive toxicity effects later in life, including increased incidence of hypospadias after puberty (Mclntyre et al. 2001, Bowman et al. 2003, Welsh et al. 2008, Christiansen et al. 2009). When the alterations in AGD and NR are relatively small, as in this study, we would not expect to see marked effects on genital malformations or reduced phallus length, as seen with more severe reductions in AGD (Welsh et al. 2008, Christiansen et al. 2009). However, even small changes in these endpoints, if significantly different from concurrent controls, should be taken as a sign of disrupted sex hormone action during critical periods of reproductive development.

Furthermore, investigation of AGD has also proven to be a relevant endpoint with regards to disrupted sexual differentiation in humans, as recent epidemiological studies indicate that boys with hypospadias or cryptorchidism have shorter AGDs compared with boys with normal genitalia (Hsieh et al. 2008) and shorter male AGDs have been reported in humans infants prenatally exposed to high levels of phthalates (Swan et al. 2005, Swan 2008).

The observed reductions in ventral prostate weights in 16-day-old males from the TotalMix450, the AAMix200, and the AAMix450 groups indicated that the anti-androgens contributed markedly to the effect of the total mixture, whereas estrogens and paracetamol did not appear to alter ventral prostate weights at this age. This was as expected, as reductions in prepubertal ventral prostate weights have been described after perinatal exposure to anti-androgens (Metzdorff et al. 2007, Christiansen et al. 2009), while the same effect of estrogens has been seen only at high dose levels. The estrogenic u.v.-filter OMC, which is present in the EMix and TotalMix, was found to reduce prepubertal ventral prostate weight only at a dose of $1000 \mathrm{mg} / \mathrm{kg} \mathrm{BW}$ per day, which is 19 times higher than the dose applied in the highest mixture groups in this study (Axelstad et al. 2011). In adult animals, estrogenic compounds may increase or decrease prostate weights depending on dose level (vom Saal et al. 1997, Putz et al. 2001). Although weights of $L A B C$ appeared lower in all TotalMix groups, the $L A B C$ weight reduction was only statistically significant in the AAMix450 and the PM groups. LABC weights are known to be sensitive to perinatal antiandrogen exposure (Metzdorff et al. 2007, Christiansen et al. 2009). No significant difference between LABC 
weights of the TotalMix450 and the AAMix450 groups was identified, and the presence of the four chosen estrogenic compounds in the TotalMix, did not appear to influence the results at the tested doses.

Interestingly, there were differences between TotalMix450 and AAMix450 groups regarding testis weight, as pups exposed to AAMix450 had larger testes than pups exposed to TotalMix450, although no significant differences were seen in controls. It may be speculated that a slight increasing effect in the anti-androgens on testis weight was counteracted by a slight depressing effect in the estrogenic components of the TotalMix on testis weight. A previous study showed increasing testis weights in 16-day-old male offspring following perinatal exposure to a mixture of anti-androgenic pesticides (Jacobsen et al. 2012), and a study on the estrogenic u.v.-filter OMC showed reduced testis weight in males of the same age, following perinatal exposure (Axelstad et al. 2011).

Increased ovary weights observed in TotalMix-450 and AAMix-450 groups could indicate an influence of anti-androgens on ovarian development. However, comparison of the present results with the results from the exploratory study indicated no exposure-related differences between ovaries from control and exposed animals, which indicates that these results were chance findings.

The results of this study confirm that highly exposed women of reproductive age may not be protected sufficiently against the combined effects of chemicals that affect the hormonal milieu required for proper sexual differentiation. The PODI calculations indicated that doses 62-fold higher than the adjusted high-end human exposure estimates should be tolerated by the rat without signs of disruption of sexual differentiation. In our previous study (Christiansen et al. 2012), adverse effects on NR were demonstrated at 150-fold higher doses, whereas this study showed clear effects on AGD, $\mathrm{NR}$, and prostate weight at 200-fold higher doses.

The concerns about insufficient margins of safety for highly exposed population groups have to be discussed in the context of accessible exposure estimates, and in the light of the limitations of the study and the constraints that we faced when selecting the chemicals for the study. As background exposure to possible endocrine-disrupting chemicals present in polycarbonate water bottles, cages, and in nesting material was not controlled in this study, a background exposure to, e.g. BPA is possible. Such exposure is, however, in our opinion not very likely to have made a measurable contribution in the exposed groups, as the doses of BPA present in the mixtures were between $0.15 \mathrm{mg} / \mathrm{kg}$ per day (TotalMix100 group) and $0.675 \mathrm{mg} / \mathrm{kg}$ per day (TotalMix450 group), which is far higher than the doses estimated from migration of BPA from polycarbonate bottles into drinking water (Le et al. 2008). Based on these data, we estimate (worst case) that migration to drinking water might have resulted in BPA doses of around $1 \%$ of those used in the mixtures. However, the possible background exposure to BPA could have increased variation in the control group, thereby reducing the sensitivity of the study.

A further premise was that certain populations might experience high exposures to all of the selected chemicals simultaneously. At the time the mixture was designed, only very limited data on co-occurrence of several chemicals in one and the same individuals were available. However, recently published data from biomonitoring studies indicate that co-occurrence of high levels of environmental contaminants like phthalates, phenols, and parabens does occur, as high individual exposures to one chemical was shown often to be associated with high exposure to the other measured chemicals (Frederiksen et al. 2013a, 2013b). These data indicate that it is conceivable that certain individuals could have higher than average simultaneous exposure to a large part of the chemicals used in this study.

As also discussed in Christiansen et al. (2012), knowledge gaps concerning the in vivo effects of other candidate compounds prevented inclusion of a wider range of chemicals in the mixture. Quantitative Structure-Activity Relationship (QSAR) analyses have predicted that $\sim 10 \%$ of the 30000 chemicals listed in the European Inventory of Existing Commercial Chemical Substances (EINECS) display AR antagonism (Vinggaard et al. 2008, Jensen et al. 2011b). Therefore, the chosen chemicals cannot be regarded as being entirely a representative of the spectrum of combined exposures encountered by human populations. It is likely that the inclusion of a wider range of chemicals would have led to more pronounced disrupting effects and correspondingly lower margins of safety. However, it should be noted that human exposure to many of these agents is intermittent, and not continuous, as in this experiment. The average human exposures may therefore be lower, but as peak exposures during vulnerable periods are critical during fetal reproductive development, then information about average exposures may be of limited value in predicting risks.

The traditional focus of risk assessment on single chemicals is shifting toward considering combination effects (Christiansen et al. 2012). Our work shows that such efforts can be supported by using the PODI method, perhaps even without conducting time-consuming and costly animal experiments. The present data demonstrate that exposure of the developing mammal to a mixture of estrogenic and anti-androgenic chemicals can affect sexual development. As adverse reproductive effects were observed at mixtures reflecting 200 times high-end human exposure, which is relatively close to the safety margin covered by the regulatory uncertainty factor of 100 , the results suggest that highly exposed human population groups may not be sufficiently protected against mixtures of endocrine-disrupting chemicals. Further investigation of reproductive effects in adult and 
senescent offspring, as well as studies on mammary gland differentiation, gene expression analysis in the developing brain, and effects on neurobehavioral development may clarify if adverse endocrine-disrupting effects can be seen at 100-fold high-end human exposure levels.

\section{Supplementary data}

This is linked to the online version of the paper at http://dx.doi. org/10.1530/REP-13-0447.

\section{Declaration of interest}

The authors declare that there is no conflict of interest that could be perceived as prejudicing the impartiality of the research reported.

\section{Funding}

This article is based on work presented at the 7th Copenhagen Workshop on Endocrine Disrupters, which was supported by the Danish Ministry of the Environment - Environmental Protection Agency. Publication of this special issue was supported by the Society for Reproduction and Fertility. The authors, Marta Axelstad, Sofie Christiansen, Julie Boberg, Pernille Rosenskjold Jacobsen, Louise Krag Isling and Ulla Hass have a relationship with the Danish Ministry of the Environment - Environmental Protection Agency as they all give independent scientifically based advice to the Danish EPA. This is financed via an agreement between the Institute and the Danish EPA. Danish EPA is a governmental institution and as such a non-profit organization. The authors' participation in COW was also financed by this funding from Danish EPA. Dr Andreas Kortenkamp and Martin Scholze have no financial relationship with the Danish EPA. None of the authors have a relationship with the Society for Reproduction and Fertility. This work has been supported by a grant from the European Commission, 7th Framework Programme, CONTAMED (Contaminant mixtures and human reproductive health - novel strategies for health impact and risk assessment of endocrine disrupters), grant agreement no.: 212502) and by funding from the Danish Environmental Protection Agency.

\section{Acknowledgements}

Dorte Lykkegaard Korsbech, Lillian Sztuk, Heidi Letting, Birgitte Møller Plesning, Ulla El-Baroudy, Vibeke Kjær, Sarah Grundt Simonsen, Eva Ferdinansen and Elise Navntoft are thanked for their excellent technical assistance.

\section{References}

Albert $O$, Desdoits-Lethimonier $C$, Lesné L, Legrand A, Guillé $F$, Bensalah K, Dejucq-Rainsford N \& Jégou B 2013 Paracetamol, aspirin and indomethacin display endocrine disrupting properties in the adult human testis in vitro. Human Reproduction 28 1890-1898. (doi:10.1093/humrep/det112)

Andrade AJ, Grande SW, Talsness CE, Gericke C, Grote K, Golombiewski A, Sterner-Lock A \& Chahoud I 2006 A dose response study following in utero and lactational exposure to di-(2-ethylhexyl) phthalate (DEHP): reproductive effects on adult male offspring rats. Toxicology 228 85-97. (doi:10.1016/j.tox.2006.08.020)

Axelstad M, Boberg J, Hougaard KS, Christiansen S, Jacobsen PR, Mandrup KR, Nellemann C, Lund SP \& Hass U 2011 Effects of preand postnatal exposure to the UV-filter octyl methoxycinnamate (OMC) on the reproductive, auditory and neurological development of rat offspring. Toxicology and Applied Pharmacology 250 278-290. (doi:10.1016/j.taap.2010.10.031)

Boberg J, Axelstad M, Christiansen S, Isling LK, Geyic G, Mandrup K \& Hass U 2013 Butylparaben alters anogenital distance, mammary development, and reproductive organ weights in perinatally exposed rats. Poster at 7th Copenhagen Workshop on Endocrine Disrupters, Copenhagen, Denmark, May 2013.

Bowman CJ, Barlow NJ, Turner KJ, Wallace DG \& Foster PM 2003 Effects of in utero exposure to finasteride on androgen-dependent reproductive development in the male rat. Toxicological Sciences 74 393-406. (doi:10.1093/toxsci/kfg128)

Bretz F, Pinheiro JC \& Branson M 2005 Combining multiple comparisons and modelling techniques in dose-response studies. Biometrics $\mathbf{6 1}$ 738-748. (doi:10.1111/j.1541-0420.2005.00344.x)

Carruthers CM \& Foster PM 2005 Critical window of male reproductive tract development in rats following gestational exposure to di- $n$-butyl phthalate. Birth Defects Research. Part B, Developmental and Reproductive Toxicology 74 277-285. (doi:10.1002/bdrb.20050)

Christiansen S, Scholze M, Dalgaard M, Vinggaard AM, Axelstad M, Kortenkamp A \& Hass U 2009 Synergistic disruption of external male sex organ development by a mixture of four anti-androgens. Environmental Health Perspectives 117 1839-1846. (doi:10.1289/ehp.0900689)

Christiansen S, Boberg J, Axelstad M, Dalgaard M, Vinggaard AM, Metzdorff SB \& Hass U 2010 Low-dose perinatal exposure to di(2-ethylhexyl) phthalate induces anti-androgenic effects in male rats. Reproductive Toxicology 30 313-321. (doi:10.1016/j.reprotox.2010.04.005)

Christiansen S, Kortenkamp A, Axelstad M, Boberg J, Scholze M, Jacobsen PR, Faust $M$, Lichtensteiger W, Schlumpf M, Burdorf $A$ et al. 2012 Mixtures of endocrine disrupting contaminants modelled on human high end exposures: an exploratory study in rats. International Journal of Andrology 35 303-316. (doi:10.1111/j.1365-2605.2011. 01242.x)

Christiansen S, Axelstad M, Boberg J, Vinggaard AM, Pedersen GA \& Hass U 2014 Low dose effects of BPA on early sexual development of male and female rats. Reproduction $\mathbf{1 4 7}$ (this issue).

Cowin PA, Gold E, Aleksova J, O'Bryan MK, Foster PM, Scott HS \& Risbridger GP 2010 Vinclozolin exposure in utero induces postpubertal prostatitis and reduces sperm production via a reversible hormoneregulated mechanism. Endocrinology 151 783-792. (doi:10.1210/en. 2009-0982)

Durrer S, Ehnes C, Fuetsch M, Maerkel K, Schlumpf M \& Lichtensteiger W 2007 Estrogen sensitivity of target genes and expression of nuclear receptor coregulators in rat prostate after pre- and postnatal exposure to the ultraviolet filter 4-methylbenzylidene camphor. Environmental Health Perspectives 115 42-50.

EFSA -European Food Safety Authority 2010 Scientific opinion on bisphenol A: evaluation of a study investigating its neurodevelopmental toxicity, review of recent scientific literature on its toxicity and advice on the Danish risk assessment of bisphenol A. EFSA Journal 81829.

Ermler S, Scholze M \& Kortenkamp A 2010 The sensitivity of the MDS-kb2 cell in vitro assay in detecting anti-androgenic chemicals - identification of sources of variability and estimation of statistical power. Toxicology in Vitro 24 1845-1853. (doi:10.1016/j.tiv.2010.05.007)

Ermler S, Scholze M \& Kortenkamp A 2011 The suitability of concentration addition for predicting the effects of multi-component mixtures of up to 17 anti-androgens with varied structural features in an in vitro AR antagonist assay. Toxicology and Applied Pharmacology 257 189-197. (doi:10.1016/j.taap.2011.09.005)

Frederiksen $H$, Nielsen JK, Mørck TA, Hansen PW, Jensen JF, Nielsen $O$, Andersson AM \& Knudsen LE 2013a Urinary excretion of phthalate metabolites, phenols and parabens in rural and urban Danish motherchild pairs. International Journal of Hygiene and Environmental Health 216 772-783. (doi:10.1016/j.ijheh.2013.02.006)

Frederiksen H, Aksglaede L, Sorensen K, Nielsen O, Main KM, Skakkebaek NE, Juul A \& Andersson AM 2013b Bisphenol A and other 
phenols in urine from Danish children and adolescents analyzed by isotope diluted TurboFlow-LC-MS/MS. International Journal of Hygiene and Environmental Health 216 710-720. (doi:10.1016/j.ijheh.2013. 01.007)

Gallavan RH, Holson JF, Stump DG, Knapp JF \& Reynolds VL 1999 Interpreting the toxicologic significance of alterations in anogenital distance: potential for confounding effects of progeny body weights. Reproductive Toxicology 13 383-390. (doi:10.1016/S0890-6238 (99)00036-2)

Gray LE Jr, Ostby JS \& Kelce WR 1994 Developmental effects of an environmental anti-androgen: the fungicide vinclozolin alters sex differentiation of the male rat. Toxicology and Applied Pharmacology 129 46-52. (doi:10.1006/taap.1994.1227)

Gray LE Jr, Ostby J, Monosson E \& Kelce WR 1999 Environmental antiandrogens: low doses of the fungicide vinclozolin alter sexual differentiation of the male rat. Toxicology and Industrial Health $\mathbf{1 5}$ 48-64. (doi:10.1191/074823399678846646)

Gupta U, Malhotra N, Varma SK \& Chaudhury RR 1981 Effect of intrauterine administration of antiprostaglandin drugs on implantation in the rat. Contraception 24 283-288. (doi:10.1016/0010-7824 (81) $90041-X)$

Hass U, Scholze M, Christiansen S, Dalgaard M, Vinggaard AM, Axelstad M, Metzdorff SB \& Kortenkamp A 2007 Combined exposure to anti-androgens exacerbates disruption of sexual differentiation in the rat. Environmental Health Perspectives 115 122-128. (doi:10.1289/ ehp.9360)

Hass U, Boberg J, Christiansen S, Jacobsen PR, Vinggaard AM, Taxvig C, Poulsen ME, Herrmann SS, Jensen BH, Petersen A et al. 2012 Adverse effects on sexual development in rat offspring after low dose exposure to a mixture of endocrine disrupting pesticides. Reproductive Toxicology 34 261-274. (doi:10.1016/j.reprotox.2012.05.090)

Hotchkiss AK, Parks-Saldutti LG, Ostby JS, Lambright C, Furr J, Vandenbergh JG \& Gray LE Jr 2004 A mixture of the "anti-androgens" linuron and butyl benzyl phthalate alters sexual differentiation of the male rat in a cumulative fashion. Biology of Reproduction $\mathbf{7 1}$ 1852-1861. (doi:10.1095/biolreprod.104.031674)

Hotchkiss AK, Rider CV, Blystone CR, Wilson VS, Hartig PC, Ankley GT, Foster PM, Gray CL \& Gray LE 2008 Fifteen years after "Wingspread" environmental endocrine disrupters and human and wildlife health: where we are today and where we need to go. Toxicological Sciences 105 235-259. (doi:10.1093/toxsci/kfn030)

Howdeshell KL, Wilson VS, Furr J, Lambright CR, Rider CV, Blystone CR, Hotchkiss AK \& Gray LE Jr 2008 A mixture of five phthalate esters inhibits fetal testicular testosterone production in the Sprague-Dawley rat in a cumulative, dose-additive manner. Toxicological Sciences 105 153-165. (doi:10.1093/toxsci/kfn077)

Hsieh MH, Breyer BN, Eisenberg ML \& Baskin LS 2008 Associations among hypospadias, cryptorchidism, anogenital distance, and endocrine disruption. Current Urology Reports 9 137-142. (doi:10.1007/s11934008-0025-0)

Isling LK, Boberg J, Jacobsen PR, Mandrup KR, Axelstad M, Christiansen S, Vinggaard AM, Taxvig C, Kortenkamp A \& Hass U 2014 Late life effects on rat reproductive system after developmental exposure to mixtures of endocrine disrupters. Reproduction 147 (this issue).

Jacobsen PR, Axelstad M, Boberg J, Isling LK, Christiansen S, Mandrup KR, Berthelsen LO, Vinggaard AM \& Hass U 2012 Persistent developmental toxicity in rat offspring after low dose exposure to a mixture of endocrine disrupting pesticides. Reproductive Toxicology 34 237-250. (doi:10.1016/j.reprotox.2012.05.099)

Jensen MS, Rebordosa C, Thulstrup AM, Toft G, Sørensen HT, Bonde JP, Henriksen TB \& Olsen J 2010 Maternal use of acetaminophen, ibuprofen, and acetylsalicylic acid during pregnancy and risk of cryptorchidism. Epidemiology 21 779-785. (doi:10.1097/EDE. Ob013e3181f20bed)

Jensen MS, Henriksen TB, Rebordosa C, Thulstrup AM, Toft G, Sørensen HT, Bonde JP \& Olsen J 2011 a Analgesics during pregnancy and cryptorchidism: additional analyses. Epidemiology 22 610-612. (doi:10.1097/EDE.0b013e31821eca69)

Jensen GE, Nikolov NG, Wedebye EB, Ringsted T \& Niemelä JR 2011b QSAR models for anti-androgenic effect - a preliminary study. SAR and QSAR in Environmental Research 22 35-49. (doi:10.1080/1062936X. 2010.528981)
Kang KS, Che JH, Ryu DY, Kim TW, Li GX \& Lee YS 2002 Decreased sperm number and motile activity on the F1 offspring maternally exposed to butyl p-hydroxybenzoic acid (butyl paraben). Journal of Veterinary Medical Science 64 227-235. (doi:10.1292/jvms.64.227)

Kavlock R \& Cummings A 2005 Mode of action: inhibition of androgen receptor function - vinclozolin-induced malformations in reproductive development. Critical Reviews in Toxicology 35 721-726. (doi:10.1080/ 10408440591007377)

Kelce WR, Monosson E, Gamcsik MP, Laws SC \& Gray LE Jr 1994 Environmental hormone disruptors: evidence that vinclozolin developmental toxicity is mediated by anti-androgenic metabolites. Toxicology and Applied Pharmacology 126 276-285. (doi:10.1006/taap.1994. 1117)

Kelce WR, Stone CR, Laws SC, Gray LE Jr, Kemppainen JA \& Wilson EM 1995 Persistent DDT metabolite $p, p^{\prime}$-DDE is a potent androgen receptor antagonist. Nature 375 581-585. (doi:10.1038/375581a0)

Kelce WR, Lambright CR, Gray LE Jr \& Roberts KP 1997 Vinclozolin and $p, p^{\prime}$-DDE alter androgen-dependent gene expression: in vivo confirmation of an androgen receptor-mediated mechanism. Toxicology and Applied Pharmacology 142 192-200. (doi:10.1006/taap.1996.7966)

Kristensen DM, Hass U, Lesné L, Lottrup G, Jacobsen PR, DesdoitsLethimonier C, Boberg J, Petersen JH, Toppari J, Jensen TK et al. 2011 Intrauterine exposure to mild analgesics is a risk factor for development of male reproductive disorders in human and rat. Human Reproduction 26 235-244. (doi:10.1093/humrep/deq323)

Kristensen DM, Lesné L, Le Fol V, Desdoits-Lethimonier C, DejucqRainsford N, Leffers H \& Jégou B 2012 Paracetamol (acetaminophen), aspirin (acetylsalicylic acid) and indomethacin are anti-androgenic in the rat foetal testis. International Journal of Andrology 35 377-384. (doi:10.1111/j.1365-2605.2012.01282.x)

Laier P, Metzdorff SB, Borch J, Hagen ML, Hass U, Christiansen S, Axelstad M, Kledal T, Dalgaard M, McKinnell C et al. 2006 Mechanisms of action underlying the anti-androgenic effects of the fungicide prochloraz. Toxicology and Applied Pharmacology 213 160-171. (doi:10.1016/j.taap.2005.10.013)

Le HH, Carlson EM, Chua JP \& Belcher SM 2008 Bisphenol A is released from polycarbonate drinking bottles and mimics the neurotoxic actions of estrogen in developing cerebellar neurons. Toxicology Letters $\mathbf{1 7 6}$ 149-156. (doi:10.1016/j.toxlet.2007.11.001)

Mazaud-Guittot S, Nicolaz CN, Desdoits-Lethimonier C, Coiffec I, Maamar MB, Balaguer P, Kristensen DM, Chevrier C, Lavoué V, Poulain P et al. 2013 Paracetamol, aspirin, and indomethacin induce endocrine disturbances in the human fetal testis capable of interfering with testicular descent. Journal of Clinical Endocrinology and Metabolism 98 E1757-E1767. (doi:10.1210/jc.2013-2531)

McIntyre BS, Barlow NJ, Wallace DG, Maness SC, Gaido KW \& Foster PM 2000 Effects of in utero exposure to linuron on androgen-dependent reproductive development in the male $\mathrm{Crl}: \mathrm{CD}(\mathrm{SD}) \mathrm{BR}$ rat. Toxicology and Applied Pharmacology 167 87-99. (doi:10.1006/taap.2000.8998)

McIntyre BS, Barlow NJ \& Foster PM 2001 Androgen-mediated development in male rat offspring exposed to fluta-mide in utero: permanence and correlation of early postnatal changes in anogenital distance and nipple retention with malformations in androgen-dependent tissues. Toxicological Sciences 62 236-249. (doi:10.1093/toxsci/62.2.236)

McIntyre BS, Barlow NJ \& Foster PM 2002 Male rats exposed to linuron in utero exhibit permanent changes in anogenital distance, nipple retention, and epididymal malformations that result in subsequent testicular atrophy. Toxicological Sciences 65 62-70. (doi:10.1093/ toxsci/65.1.62)

Metzdorff SB, Dalgaard M, Christiansen S, Axelstad M, Hass U, Kiersgaard MK, Scholze M, Kortenkamp A \& Vinggaard AM 2007 Dysgenesis and histological changes of genitals and perturbations of gene expression in male rats after in utero exposure to anti-androgen mixtures. Toxicological Sciences 98 87-98. (doi:10.1093/toxsci/kfm079)

Mylchreest E, Cattley RC \& Foster PM 1998 Male reproductive tract malformations in rats following gestational and lactational exposure to di(n-butyl) phthalate: an anti-androgenic mechanism? Toxicological Sciences 43 47-60.

Mylchreest E, Wallace DG, Cattley RC \& Foster PM 2000 Dose-dependent alterations in androgen-regulated male reproductive development in rats exposed to di(n-butyl) phthalate during late gestation. Toxicological Sciences 55 143-151. (doi:10.1093/toxsci/55.1.143) 
Ostby J, Kelce WR, Lambright C, Wolf CJ, Mann P \& Gray LE Jr 1999 The fungicide procymidone alters sexual differentiation in the male rat by acting as an androgen-receptor antagonist in vivo and in vitro. Toxicology and Industrial Health 15 80-93.

Parks LG, Ostby JS, Lambright CR, Abbott BD, Klinefelter GR, Barlow N \& Gray LE Jr 2000 The plasticizer diethylhexyl phthalate induces malformations by decreasing fetal testosterone synthesis during sexual differentiation in the male rat. Toxicological Sciences 58 339-349. (doi:10.1093/toxsci/58.2.339)

Putz O, Schwartz CB, Kim S, LeBlanc GA, Cooper RL \& Prins GS 2001 Neonatal low- and highdose exposure to estradiol benzoate in the male rat: I. Effects on the prostate gland. Biology of Reproduction 65 1496-1505. (doi:10.1095/biolreprod65.5.1496)

Rider CV, Furr J, Wilson VS \& Gray LE Jr 2008 A mixture of seven antiandrogens induces reproductive malformations in rats. International Journal of Andrology 31 249-262. (doi:10.1111/j.1365-2605.2007.00859.x)

Rider CV, Furr JR, Wilson VS \& Gray LE Jr 2010 Cumulative effects of in utero administration of mixtures of reproductive toxicants that disrupt common target tissues via diverse mechanisms of toxicity. International Journal of Andrology 33 443-462. (doi:10.1111/j.1365-2605.2009. 01049.x)

vom Saal FS, Timms BG, Montano MM, Palanza P, Thayer KA, Nagel SC, Dhar MD, Ganjam VK, Parmigiani S \& Welshons Wv 1997 Prostate enlargement in mice due to fetal exposure to low doses of estradiol or diethylstilbestrol and opposite effects at high doses. PNAS 94 2056-2061. (doi:10.1073/pnas.94.5.2056)

Salian S, Doshi T \& Vanage G 2009 Perinatal exposure of rats to bisphenol A affects the fertility of male offspring. Life Sciences 85 742-752. (doi:10.1016/j.lfs.2009.10.004)

Schlumpf $M$, Cotton B, Conscience $M$, Haller V, Steinmann B \& Lichtensteiger W 2001 In vitro and in vivo estrogenicity of UV screens. Environmental Health Perspectives 109 239-244. (doi:10.1289/ehp. 01109239)

Schreurs R, Lauser P, Seinen W \& van den Burg B 2002 Estrogenic activity of UV filters determined by an in vitro reporter gene assay and an in vivo transgenic zebrafish assay. Archives of Toxicology 76 257-261. (doi:10.1007/s00204-002-0348-4)

Schultz VD, Phillips S, Sar M, Foster PM \& Gaido KW 2001 Altered gene profiles in fetal rat testes after in utero exposure to di(n-butyl) phthalate. Toxicological Sciences 64 233-242. (doi:10.1093/toxsci/64.2.233)

Silva E, Rajapakse N \& Kortenkamp A 2002 Something from "nothing" eight weak estrogenic chemicals combined at concentrations below NOECs produce significant mixture effects. Environmental Science \& Technology 36 1751-1756. (doi:10.1021/es0101227)

Snijder CA, Kortenkamp A, Steegers EA, Jaddoe VW, Hofman A, Hass U \& Burdorf A 2012 Intrauterine exposure to mild analgesics during pregnancy and the occurrence of cryptorchidism and hypospadia in the offspring: the Generation R Study. Human Reproduction 27 1191-1201. (doi:10.1093/humrep/der474)

Swan SH 2008 Environmental phthalate exposure in relation to reproductive outcomes and other health endpoints in humans. Environmental Research 108 177-184. (doi:10.1016/j.envres.2008.08.007)

Swan SH, Main KM, Liu F, Stewart SL, Kruse RL, Calafat AM, Mao CS, Redmon JB, Ternand CL, Sullivan S et al. 2005 Study for Future Families
Research Team. Decrease in anogenital distance among male infants with prenatal phthalate exposure. Environmental Health Perspectives 113 1056-1061. (doi:10.1289/ehp.8100)

Taxvig C, Hass U, Axelstad M, Dalgaard M, Boberg J, Andersen HR \& Vinggaard AM 2007 Endocrine-disrupting activities in vivo of the fungicides tebuconazole and epoxiconazole. Toxicological Sciences 100 464-473. (doi:10.1093/toxsci/kfm227)

Tinwell H, Lefevre PA, Moffat GJ, Burns A, Odum J, Spurway TD, Orphanides G \& Ashby J 2002 Confirmation of uterotrophic activity of 3(4-methylbenzylidene)camphor in the immature rat. Environmental Health Perspectives 110 533-536. (doi:10.1289/ehp.02110533)

Vinggaard AM, Christiansen S, Laier P, Poulsen ME, Breinholt V, Jarfelt K, Jacobsen H, Dalgaard M, Nellemann C \& Hass U 2005 Perinatal exposure to the fungicide prochloraz feminizes the male rat offspring. Toxicological Sciences 85 886-897. (doi:10.1093/toxsci/ kfi150)

Vinggaard AM, Hass U, Dalgaard M, Andersen HR, Bonefeld-Jorgensen E, Christiansen S, Laier P \& Poulsen ME 2006 Prochloraz: an imidazole fungicide with multiple mechanisms of action. International Journal of Andrology 29 186-192. (doi:10.1111/j.1365-2605.2005.00604.x)

Vinggaard AM, Niemela J, Wedebye EB \& Jensen GE 2008 Screening of 397 chemicals and development of a quantitative structure-activity relationship model for androgen receptor antagonism. Chemical Research in Toxicology 21 813-823. (doi:10.1021/tx7002382)

Welsh M, Saunders PT, Fisken M, Scott HM, Hutchison GR, Smith LB \& Sharpe RM 2008 Identification in rats of a programming window for reproductive tract masculinization, disruption of which leads to hypospadias and cryptorchidism. Journal of Clinical Investigation 118 1479-1490. (doi:10.1172/JCl34241)

Wilson VS, Lambright C, Furr J, Ostby J, Wood C, Held G \& Gray LE Jr 2004 Phthalate ester-induced gubernacular lesions are associated with reduced insl3 gene expression in the fetal rat testis. Toxicology Letters 146 207-215. (doi:10.1016/j.toxlet.2003.09.012)

Wolf C, Lambright C, Mann P, Price M, Cooper RL, Ostby J \& Gray LE Jr 1999 Administration of potentially anti-androgenic pesticides (procymidone, linuron, iprodione, chlozolinate, $p, p^{\prime}$-DDE, and ketoconazole) and toxic substances (dibutyl- and diethylhexyl phthalate, PCB 169, and ethane dimethane sulphonate) during sexual differentiation produces diverse profiles of reproductive malformations in the male rat. Toxicology and Industrial Health 15 94-118. (doi:10.1177/ 074823379901500109)

You L, Casanova M, Archibeque-Engle S, Sar M, Fan L \& Heck HAD 1998 Impaired male sexual development in perinatal Sprague-Dawley and Long-Evans hooded rats exposed in utero and lactationally to $p, p^{\prime}$-DDE. Toxicological Sciences 45 162-173.

Received 13 September 2013

First decision 22 October 2013

Revised manuscript received 22 November 2013

Accepted 2 December 2013 